\title{
Generation and complete temporal characterization of 5-fs EUV pulses
}

\author{
Matteo Lucchini ${ }^{1,2, *}$, Giacinto D. Lucarelli ${ }^{1}$, Mario Murari ${ }^{1,2}$, Andrea Trabattoni ${ }^{3}$, Nicola \\ Fabris $^{4,5}$, Fabio Frassetto ${ }^{4}$, Sandro De Silvestri ${ }^{1,2}$, Luca Poletto $^{4}$, Mauro Nisoli ${ }^{1,2}$ \\ ${ }^{1}$ Department of Physics, Politecnico di Milano, 20133 Milano, Italy \\ ${ }^{2}$ Institute for Photonics and Nanotechnologies, IFN-CNR, 20133 Milano, Italy \\ ${ }^{3}$ Center for Free-Electron Laser Science (CFEL), DESY, 22607 Hamburg, Germany \\ ${ }^{4}$ Institute for Photonics and Nanotechnologies, IFN-CNR, 35131 Padova, Italy \\ ${ }^{5}$ Department of Information Engineering, University of Padova, 35131 Padova, Italy
}

\begin{abstract}
Extreme-ultraviolet pulses as short as $5 \mathrm{fs}$, produced by highorder harmonic generation and selected by a time-delay compensated monochromator, were fully reconstructed thanks to an advanced ptychographic technique, while most commonly used reconstruction methods fail. These pulses constitute the shortest extreme ultraviolet pulses ever achieved via a time-delay compensated monochromator.
\end{abstract}

\section{Introduction}

Few-femtosecond pulses, broadly tunable in the extreme-ultraviolet (EUV) spectral region are particularly important in many research fields: from atomic and molecular spectroscopy to the investigation of ultrafast relaxation processes in solids, from holography to free-electron laser injection [1]. The essential prerequisite for all applications is the accurate measurement of the temporal characteristics of these pulses. Many measurement schemes and phase retrieval algorithms have been employed, in particular for the characterization of attosecond pulses, but their extension to EUV few-femtosecond pulses is not trivial and has never been reported so far.

Here we demonstrate that sub-10-fs EUV pulses, produced by high-order harmonic generation (HHG) and spectrally selected by a time-delay compensated monochromator (TDCM) [2], can be fully characterized, in terms of temporal intensity and phase, by employing the ptychographic reconstruction technique [3], while other common reconstruction algorithms fail. The implementation of this reconstruction method enabled us to achieve a complete temporal characterization of EUV pulses with duration down to 5 fs, which are the shortest pulses ever generated in this spectral region by employing an EUV monochromator.

\footnotetext{
* Corresponding author: matteo.lucchini@polimi.it
} 


\section{Results and discussion}

15 -fs pulses at 800 -nm central wavelength were used to generate high-order harmonics in a gas cell filled with argon. The harmonic radiation was then coupled into a TDCM, allowing the selection of a single harmonic, while preserving its original temporal duration [2]. After the monochromator, the EUV pulses and a fraction of the driving infrared (IR) pulses were recombined by employing a close-to-collinear geometry and focused into an interaction chamber, equipped with a gas nozzle and a time-of-flight electron spectrometer. When the two pulses overlap in time and space on the gas jet, sidebands appear in the photoelectron spectrum, spectrally shifted by the IR photon energy, determined by the absorption of one harmonic photon plus absorption or emission of at least one IR photon. The number of generated sidebands depends on the IR laser intensity. Figure 1(a) shows the experimental spectrogram, measured in neon, in the case of the EUV pulse obtained by selecting the 27th harmonic (HH27).

In the case of attosecond pulses, the measurement method illustrated above is called frequency-resolved optical gating for the complete reconstruction of attosecond bursts (FROG CRAB) technique [4]. The measured spectrogram is a real and positive quantity so that phase information are lost. So far, several algorithms have been proposed and implemented to reconstruct the phase associated with spectrograms measured in the case of EUV pulses with attosecond duration [5-6]. The narrower bandwidth associated with a single harmonic and the associated loss of sub-cycle resolution, reduce the level of information redundancy in the spectrogram. For this reason, convergence is more critical and conventional algorithms like the principal component generalized projection algorithm (PCGPA) and the least squared generalized projection algorithm (LSGPA) fail in the case of reconstruction of few-femtosecond EUV pulses.
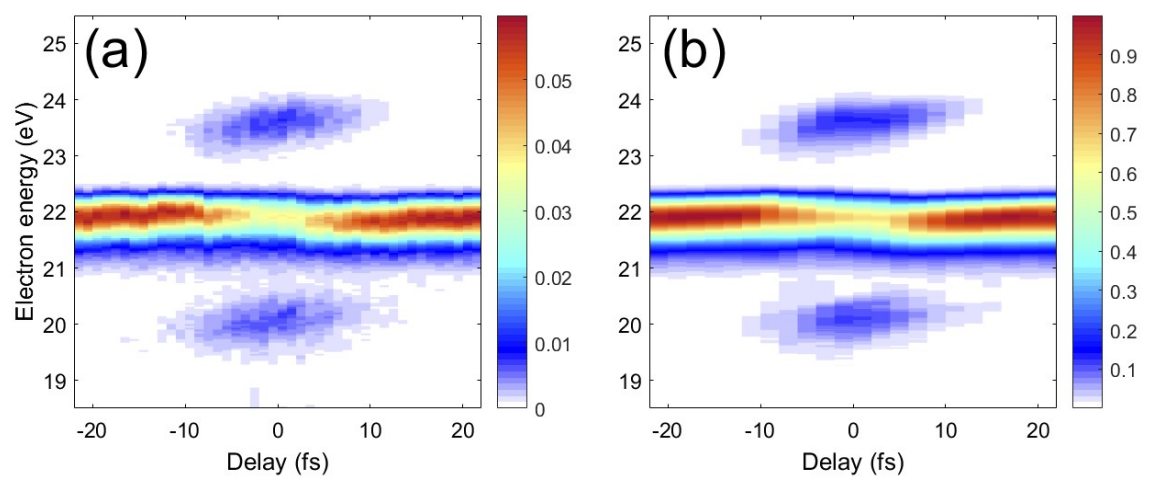

Fig. 1. (a) Spectrogram of the EUV pulse obtained by selecting the 27th harmonic, measured in neon, scanning the EUV-IR temporal delay with 1-fs steps. (b) ePIE reconstruction (2000 iterations).

The main result of this work is the demonstration that the extended ptychographic iterative engine (ePIE) is a powerful method to achieve full reconstruction of the temporal characteristics of single harmonic pulses. In contrast to the most generally used algorithms based on principal projection component, in ePIE the delay and energy axis are not linked by a Fourier transform. This relaxes the need for data interpolation. Furthermore, the code is sensitive to the absolute value of the delay and not only to the relative step size, which results in an algorithm capable to work also with non-equidistant sampling. In addition, since ePIE cycles on each individual time delay step, one complete run over the spectrogram corresponds to several algorithm iterations making it robust to white noise and quick in convergence. For these reasons, ePIE is the perfect candidate to extend the FROG CRAB method to the reconstruction of single harmonic spectrograms. 

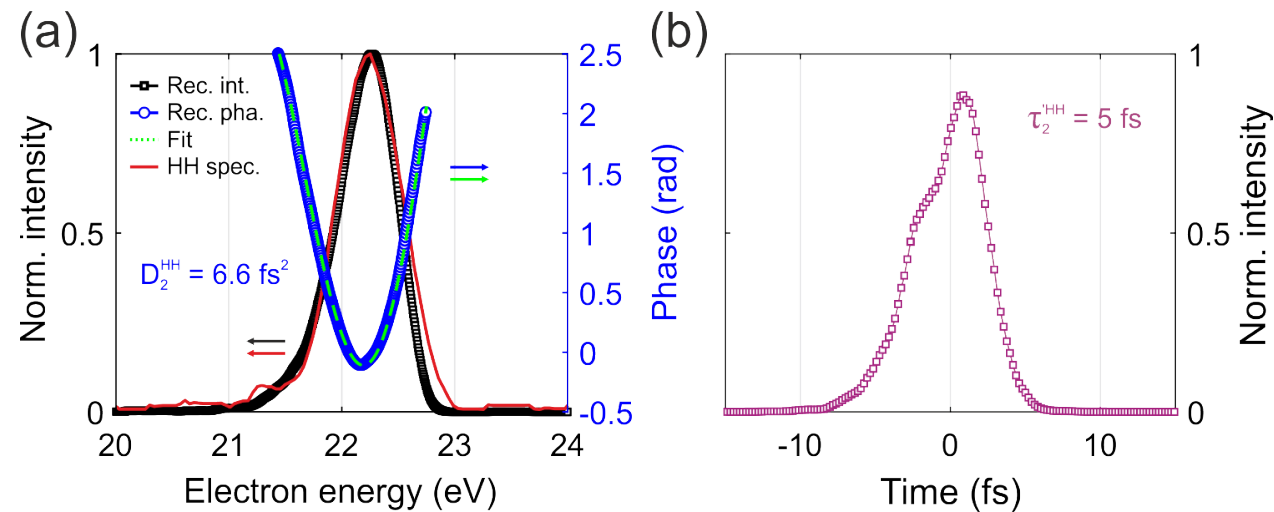

Fig. 2. (a) Reconstructed spectral intensity (black squares) and phase (blue circles) of HH27. The red solid line displays the harmonic photon spectrum measured by using an EUV spectrometer. The green dashed curve represents the $5^{\text {th }}$-order polynomial fit used to extract the group delay dispersion, $\mathrm{D}_{2}{ }^{\mathrm{HH}}$. (b) Corresponding temporal evolution of the harmonic intensity, showing a duration (FWHM) of $5 \mathrm{fs}$.

Figure 1(b) shows the ePIE reconstruction of the spectrogram reported in Fig. 1(a) for the pulse obtained by selecting the $27^{\text {th }}$ harmonic. After only 2000 iterations, the reconstructed spectrogram shows a very good agreement with the experimental one. Further evidence for the correct convergence is given by the comparison between the measured harmonic spectrum (red curve in Fig. 2(a)) and the reconstructed harmonic spectral distribution (black curve with square markers in Fig. 2(a)). The reconstructed IR pulse (not shown) has a temporal duration of $15 \mathrm{fs}$, in excellent agreement with an independent FROG measurement. The temporal evolution of the intensity profile of the harmonic pulses is reported in Fig. 2(b): the full width at half maximum duration is $5 \pm 0.5 \mathrm{fs}$, which represents, to the best of our knowledge, the shortest EUV pulse duration ever measured at the output of an EUV monochromator. The reconstructed spectral phase (blue circles in Fig. 2(a)), which was fitted by a polynomial function (green dashed curve in Fig. 2(a)), is characterized by a group delay dispersion (GDD) of $6.6 \pm 0.5 \mathrm{fs}^{2}$. The residual chirp is mainly related to the GDD introduced by the TDCM: the measured value is in the excellent agreement with the result of ray-tracing simulations, giving a residual GDD of $7 \mathrm{fs}^{2}$ for HH27. A further optimization could lead to even shorter pulses, suitable to investigate dynamics unfolding on very few fs (like spin diffusion, electron hopping and dynamical screening) which remain now largely unexplored.

\section{References}

1. A. L'Huillier, D. Descamps, A. Johansson, J. Norin, J. Mauritsson, and C. -G. Wahlström, Eur. Phys. J. D 26, 91 (2003).

2. L. Poletto, P. Villoresi, E. Benedetti, F. Ferrari, S. Stagira, G. Sansone, M. Nisoli, Opt. Lett. 32, 2897 (2007).

3. M. Lucchini, M. H. Brügmann, A. Ludwig, L. Gallmann, U. Keller and T. Feurer, Opt. Express 23, 29502 (2015).

4. Y. Mairesse and F.Quéré, Phys. Rev. A 71, 011401 (2005).

5. J. Gagnon, E. Goulielmakis and V. S. Yakovlev, Appl. Phys. B 92, 25 (2008).

6. M. Chini, S. Gilbertson, S. D. Khan and Z. Chang, Opt. Express 18, 13006 (2010). 